\title{
A Novel Broadband Printed Dipole Antenna and Its Application for TD-LTE Communications
}

\author{
Can Wang and Yuehe Ge \\ College of Information Science and Engineering, Huaqiao University, Xiamen, Fujian 361021, China \\ Correspondence should be addressed to Yuehe Ge; yuehe@ieee.org
}

Received 23 March 2014; Revised 17 July 2014; Accepted 16 August 2014; Published 4 September 2014

Academic Editor: Zhi N. Chen

Copyright (c) 2014 C. Wang and Y. Ge. This is an open access article distributed under the Creative Commons Attribution License, which permits unrestricted use, distribution, and reproduction in any medium, provided the original work is properly cited.

\begin{abstract}
Novel double-sided printed dipole antennas are proposed and investigated in this paper. Two pairs of identical T-shape metallic loadings are connected to the traditional double-sided printed dipole, enhancing the operating bandwidth. Simulations show that the proposed printed dipole without the reflecting ground plane gives a bandwidth of up to $90.7 \%$ for $\left|S_{11}\right|<-10 \mathrm{~dB}$ or $84.9 \%$ for $\left|S_{11}\right|<-15 \mathrm{~dB}$, which is significantly greater than those of the traditional printed dipole. The proposed dipole is then applied to design a dual-polarized printed dipole antenna, mounted on a ground plane. Measurements for the prototype show a bandwidth of $68.8 \%$ for $\left|S_{11}\right|<-10 \mathrm{~dB}$, covering the entire TD-LTE band. The gain is about $6-8 \mathrm{dBi}$ and the isolation is over $24 \mathrm{~dB}$ over the bandwidth.
\end{abstract}

\section{Introduction}

Various communication systems emerge in the last decade due to the rapid development of wireless communication systems to satisfy the increasing demand of service and transmission speed. Different frequency bands are employed in these systems, such as the DCS $(1.71 \mathrm{GHz}-1.88 \mathrm{GHz})$, PCS $(1.85 \mathrm{GHz}-1.99 \mathrm{GHz})$, and TD-LTE $(1.71 \mathrm{GHz}-2.69 \mathrm{GHz})$. Wideband and multiband antennas are required to cover all or parts of these frequency bands, leading to compact, lowcost, accommodating-multiple-band systems. In addition, to improve the quality and reliability of a wireless link, antenna diversity technology is used in modern communication systems. The dual-polarization antenna is one of solutions for diversity antennas.

The antenna requirements for modern communication systems include broadband, stable unidirectional patterns, low cross polarization, and high front-to-back ratio. A lot of methods have been proposed for these applications. Patch antennas with an L-shape feeding [1] provide a bandwidth of $36 \%$. A metallic dipole antenna [2], including both electric and magnetic dipoles, was developed to achieve a bandwidth of $43 \%$ and good radiation performance. Traditional printed dipole antennas with an integrated balun have advantages of wide bandwidth, symmetrical radiation patterns, structure simplicity, low cost, and ease for fabrication and have been well studied for applications of broadband communication systems. Different versions of the printed dipole have been developed to provide bandwidths of $40 \%-50 \%$ [3-6]. In [7], parasitic elements are introduced surrounding the printed dipole, leading to a bandwidth of $75.8 \%$, though the size and the cross polarization level of the antenna increase. Wideband dual-polarized antennas are also developed. Metallic dualpolarized antennas are presented in $[8,9]$, which achieve bandwidth of $65.9 \%$ and $68 \%$, respectively. A printed dualpolarized antenna with a bandwidth of over $50 \%$ for base station is proposed in [10]. All the three antennas are planar, low-profile, and high-gain.

In this paper, a novel double-sided printed dipole is proposed for wideband operations. Two pairs of identical Tshape metallic loadings are introduced to connect the two wings of the normal double-sided printed dipole to broaden the bandwidth. The traditional printed balun is applied to feed the dipole antenna. The novel printed dipole is then used to design dual-polarized nonplanar printed dipole antenna to cover the frequency band of mobile systems from $1.5 \mathrm{GHz}$ to $3 \mathrm{GHz}$. A prototype was built and tested to validate the final design. 


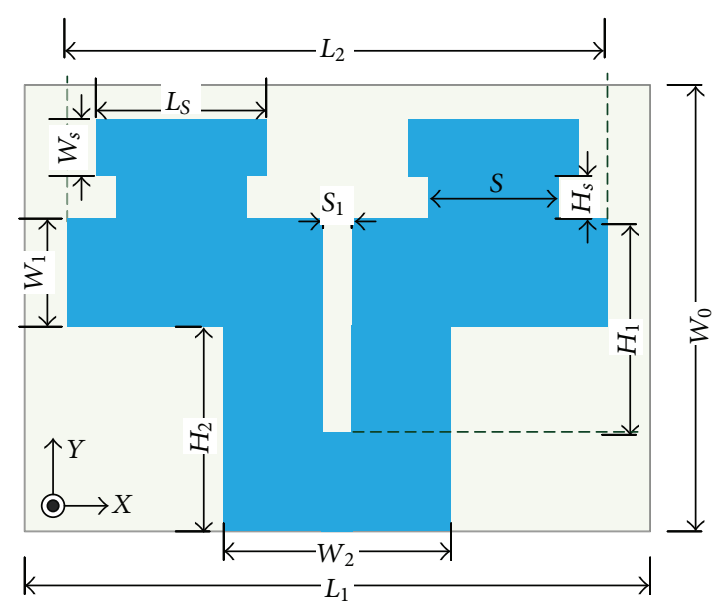

(a)

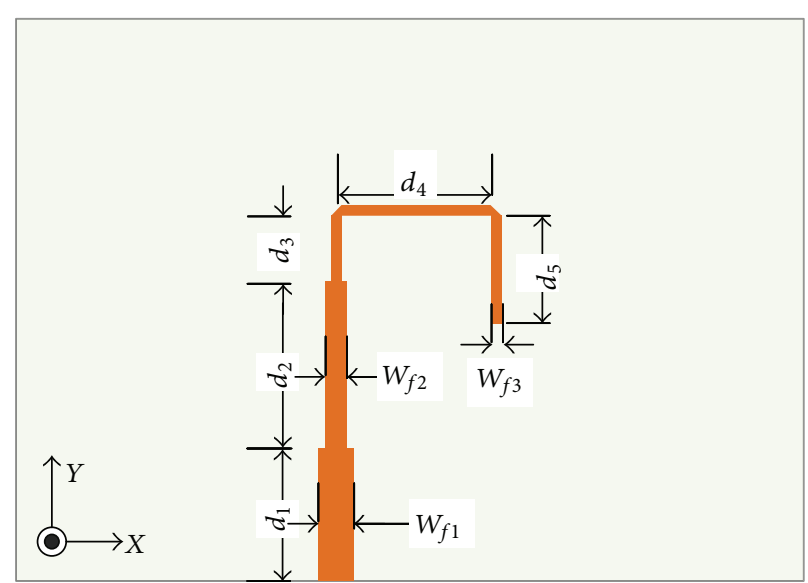

(b)

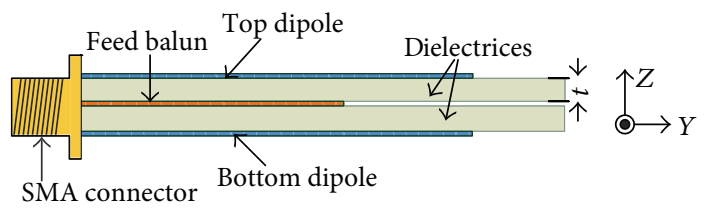

(c)

FIgURE 1: Configuration of the proposed double-sided antenna. (a) Top and bottom layers. (b) Interlayer. (c) Side view.

\section{Broadband Printed Dipole with T-Shape Loadings}

The proposed wideband printed dipole is shown in Figure 1. It consists of a double-sided printed dipole, an integrated balun, and a coaxial probe connected to an SMA connector, which are shown in Figures 1(a), 1(b), and 1(c), respectively. The double-sided printed dipole includes two identical planar dipoles printed on the two sides of a FR4/Epoxy substrate. In practice, two identical FR4/Epoxy substrates form the antenna, where the two identical planar dipoles are on the two outside surfaces and the integrated balun is printed on the inside surface of one of the FR4/Epoxy substrates. Compared to the traditional double-sided printed dipole [7], two pairs of identical metallic T-shape stubs are symmetrically connected to the printed dipole, to enhance the bandwidth. The integrated balun consists of three striplines with different characteristic impedances, namely, different widths. The transformation of the characteristic impedance can also improve the bandwidth of the antenna.

It is desired that the proposed double-sided printed dipole antenna can provide a wider bandwidth, without compromising the other performance of the traditional printed dipole, such as radiation patterns and the cross-polarization level. To validate this, a traditional (Ant 1) and a proposed (Ant 2) double-sided printed dipoles are optimized and designed, respectively. The thickness of the used FR4/Epoxy substrate is $1.6 \mathrm{~mm}$ and the total thickness of the double-sided printed dipoles is $3.2 \mathrm{~mm}$. The dimensions of the two antenna designs are listed in Table 1.

Figure 2 shows the simulated reflection coefficients, obtained from the commercial software Ansoft HFSS, for the two antenna designs. It can be seen that there are three resonant points in the operating band of the traditional printed dipole, while, by loading two identical pairs of metallic T-shape stubs on the printed dipole, the resonant points increase to four or more in the operating band and the bandwidth increases significantly. The bandwidth for the traditional printed dipole is $59.6 \%$ for $\left|S_{11}\right|<-10 \mathrm{~dB}$, from $1.58 \mathrm{GHz}$ to $2.92 \mathrm{GHz}$, and $53.6 \%$ for $\left|S_{11}\right|<-15 \mathrm{~dB}$, from 1.65 to 2.86 , while the proposed printed dipole gives a bandwidth of $90.7 \%$ for $\left|S_{11}\right|<-10 \mathrm{~dB}$, from $1.32 \mathrm{GHz}$ to $3.51 \mathrm{GHz}$, and $84.9 \%$ for $\left|S_{11}\right|<-14 \mathrm{~dB}$, from $1.39 \mathrm{GHz}$ to $3.44 \mathrm{GHz}$. The simulated results demonstrate that the bandwidth of the traditional printed dipole has been improved significantly with symmetrically loading the metallic T-shape stubs. The effect of the T-shape stubs on the impedance matching for Ant 2 is also investigated. Figure 3 shows the simulated results for Ant 2 with and without T-shape stubs, as well as those stubs with different dimensions. It can be seen that Ant 2 resonates at the higher band due to the existence of the T-shape stubs, and changing the dimensions of the stub will result in a wider bandwidth.

\section{Wideband Dual-Polarized Antenna}

Antennas with the performance of polarization diversity can reduce the effect of multipath fading and increase channel capacity in mobile communication systems. In this section, wideband dual-polarized printed dipole antenna is developed based on the proposed printed dipole in Section 2. Figure 4 shows the proposed antenna configuration. Two doublesided printed dipoles, shown in Figures 4(a) and 4(b), respectively, are placed normal to each other, to obtain 
TABLE 1: Dimensions of the optimized traditional and proposed printed dipole antennas (unit: $\mathrm{mm}$ ).

\begin{tabular}{ccccccccccccc}
\hline & \multicolumn{1}{c}{ Ant 1 } & \multicolumn{9}{c}{ Ant 2 } \\
\hline$L_{1}$ & 70 & $d_{1}$ & 5 & $W_{f 2}$ & 1.5 & $L_{1}$ & 88 & $d_{1}$ & 9 & $W_{f 2}$ & 1.4 \\
$L_{2}$ & 60 & $d_{2}$ & 13 & $W_{f 3}$ & 0.8 & $L_{2}$ & 72 & $d_{2}$ & 12 & $W_{f 3}$ & 0.6 \\
$H_{1}$ & 30 & $d_{3}$ & 9.5 & $t$ & 1.6 & $H_{1}$ & 33 & $d_{3}$ & 9 & $t$ \\
$H_{2}$ & 26 & $d_{4}$ & 8.4 & & & $H_{2}$ & 28 & $d_{4}$ & 15 & $S$ & 7 \\
$W_{0}$ & 45 & $d_{5}$ & 14.5 & & & $W_{0}$ & 54 & $d_{5}$ & 8 & $W_{s}$ & 8 \\
$W_{1}$ & 14 & $S_{1}$ & 4 & & & $W_{1}$ & 18 & $S_{1}$ & 5.2 & $L_{s}$ & 18 \\
$W_{2}$ & 30 & $W_{f 1}$ & 2.6 & & & $W_{2}$ & 33 & $W_{f 1}$ & 2 & $H_{s}$ \\
\hline
\end{tabular}

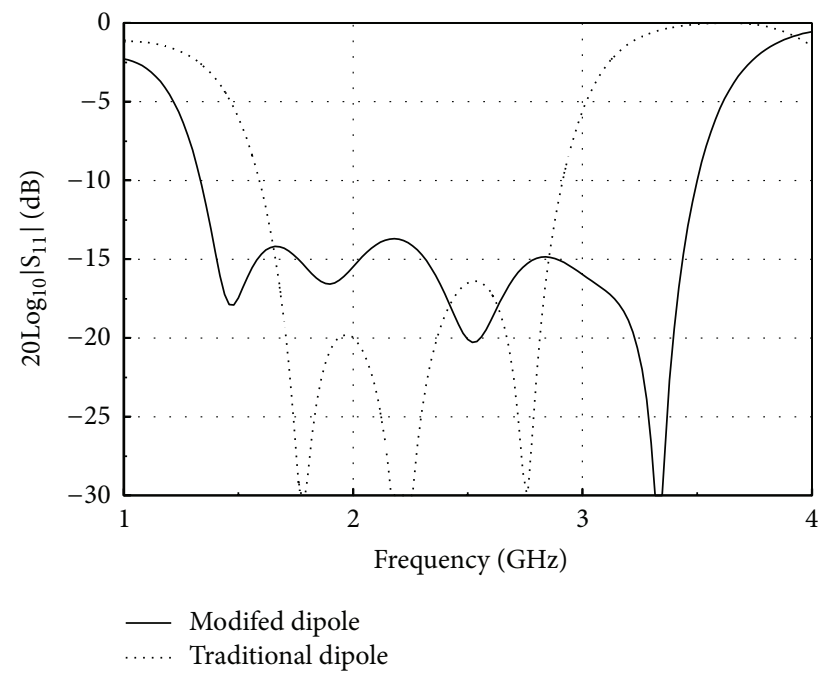

FIGURE 2: Simulated reflection coefficients.

dual-polarization performance. Each dipole consists of two identical FR4/Epoxy substrates. As can be seen, the dipole in Figure 4(a) has a shorter balun and a slot cut on the upper part of the substrate, while that in Figure 4(b) has a longer balun and a slot cut on the lower part of the substrate. Thus they are able to be cross mounted and perpendicular to each other, and the final geometry of the dual-polarized printed dipole antenna is shown in Figure 4(c). A PEC ground plane is placed on the bottom to enhance the gain and front-toback ratio performance. Due to the configuration, the two integrated baluns are also cross arranged. The heights of the two baluns are selected different, to avoid the contact between them. Therefore, the two dipoles have the same dimensions in dipoles while different dimensions in feeding baluns. Although the positions of the ports of the two baluns are separated, they are too close in practice. The two ports need to be connected to SMA connectors, which are located below the ground plane, to carry out the measurements. To avoid the mounting difficulty, the striplines of the lower parts of the two baluns are deliberately bent, to add more space between them, as shown in Figures 4(a) and 4(b). The additional advantage is that the isolation between the two ports is enhanced, as detailed below.

FR4/Epoxy material with a thickness of $1.6 \mathrm{~mm}$ is used to design the dual-polarized printed dipole antenna. Therefore, the total thickness of each double-sided printed dipole is
$3.2 \mathrm{~mm}$. The ground size is $150 \mathrm{~mm} \times 150 \mathrm{~mm}$. Extensive parameter studies are carried out and the dimensions of the printed dipoles of the final design are listed in Table 2.

The designed dual-polarized antenna was fabricated and the prototype is shown in Figure 5. The simulated and measured reflection coefficients, obtained from Ansoft HFSS and network analyzer Agilent E5071C, respectively, are plotted in Figure 6. The simulated bandwidths for ports 1 and 2, determined by $\left|S_{11}\right|<-10 \mathrm{~dB}$, are $66.4 \%$, from $1.56 \mathrm{GHz}$ to $3.11 \mathrm{GHz}$, and $64.3 \%$, from $1.54 \mathrm{GHz}$ to $3 \mathrm{GHz}$, respectively. If considering $\left|S_{11}\right|<-15 \mathrm{~dB}$, the simulated bandwidths are $61.8 \%$, from $1.61 \mathrm{GHz}$ to $3.05 \mathrm{GHz}$, and $59.6 \%$, from $1.59 \mathrm{GHz}$ to $2.94 \mathrm{GHz}$, respectively. The measured bandwidths for the two ports, determined by $\left|S_{11}\right|<-10 \mathrm{~dB}$, are $68.8 \%$, from $1.61 \mathrm{GHz}$ to $3.3 \mathrm{GHz}$, and $68 \%$, from $1.66 \mathrm{GHz}$ to $3.37 \mathrm{GHz}$, respectively. All cover the TD-LTE bands. Compared to the proposed single-polarized printed dipole, the bandwidth of the dual-polarized one is narrower. The reason is that a larger PEC ground plane is applied to increase the front-to-back ratio of the radiation patterns.

The isolation of the two ports is also simulated and measured and plotted in Figure 7. As aforementioned, adding more separate space between the two ports due to bending the lower parts of the two baluns will result in better isolation. For comparison, the two baluns without bending their ends are also applied for simulation and the result is also plotted 


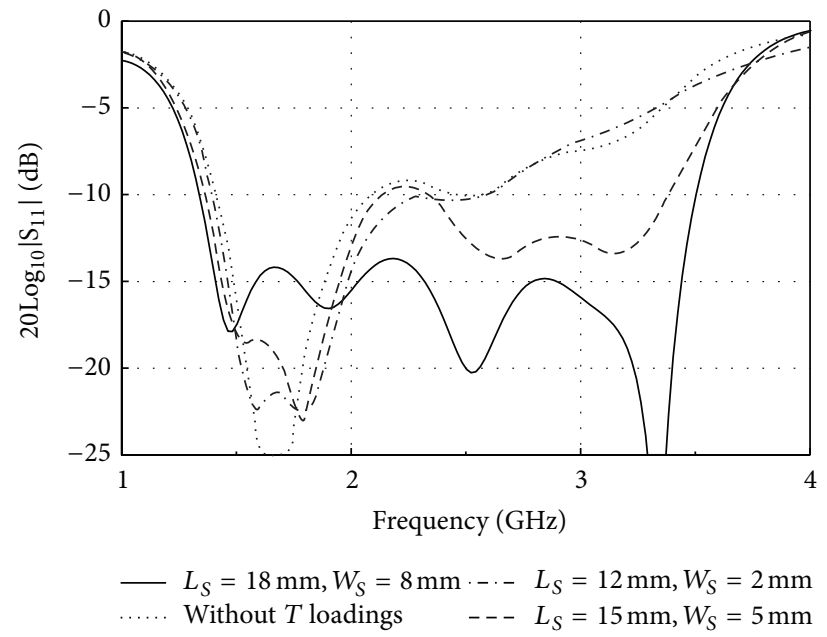

FIgURE 3: Effect of the T-shape loadings.

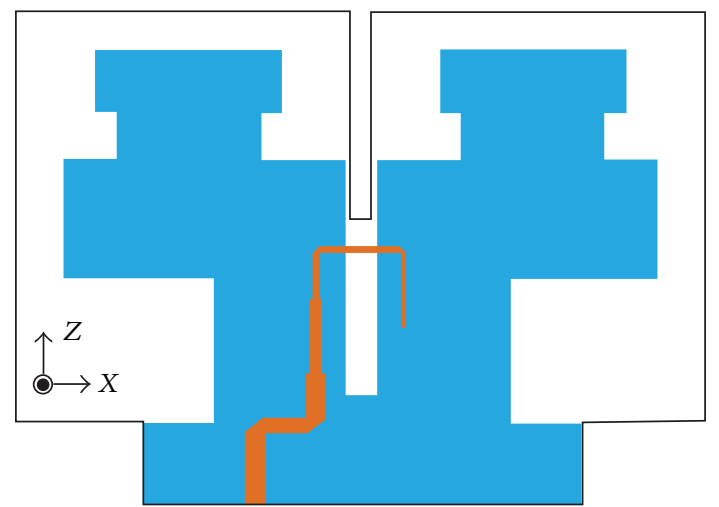

Top and bottom layers metallization Interlayer metallization

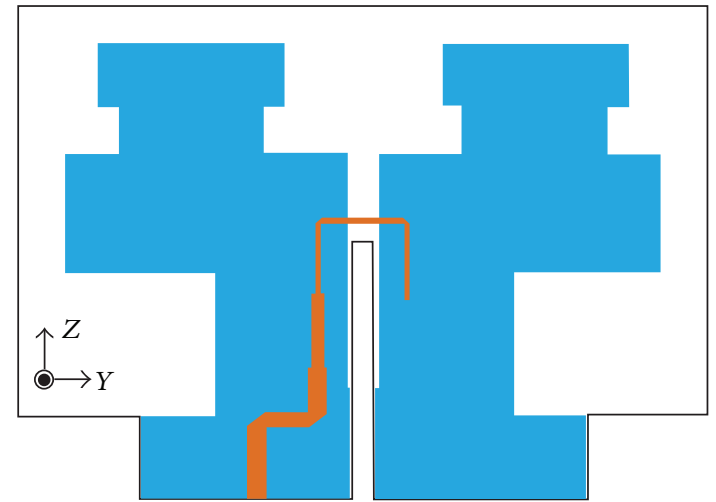

Top and bottom layers metallization

Interlayer metallization

(a)

(b)

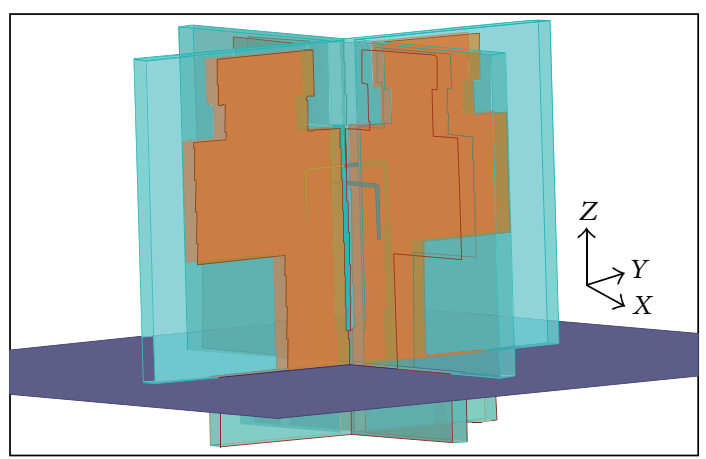

(c)

FIGURE 4: Geometry of the dual-polarized printed dipole antenna: (a) the dipole with a shorter balun and a slot on the upper part of the substrate; (b) the dipole with a longer balun and a slot on the lower part of the substrate; and (c) the geometry of the mounted dual-polarized printed dipole antenna. 
TABLE 2: Dimensions of the dual-polarized printed dipole antenna (unit: $\mathrm{mm}$ ).

\begin{tabular}{|c|c|c|c|c|c|c|c|}
\hline \multicolumn{4}{|c|}{ Antenna 1 (Figure 4(a)) } & \multicolumn{4}{|c|}{ Antenna 2 (Figure 4(b)) } \\
\hline$L_{1}$ & 88 & $d_{2}$ & 13 & $L_{1}$ & 88 & $d_{2}$ & 14 \\
\hline$L_{2}$ & 68 & $d_{3}$ & 9 & $L_{2}$ & 68 & $d_{3}$ & 11 \\
\hline$H_{1}$ & 35 & $d_{4}$ & 18 & $H_{1}$ & 35 & $d_{4}$ & 18 \\
\hline$W_{1}$ & 21 & $d_{5}$ & 9 & $W_{1}$ & 21 & $d_{5}$ & 9.5 \\
\hline$W_{2}$ & 60 & $S_{1}$ & 4.4 & $W_{2}$ & 60 & $S_{1}$ & 4.4 \\
\hline$W_{f 1}$ & 2.3 & $L_{S}$ & 21 & $W_{f 1}$ & 2.3 & $L_{S}$ & 21 \\
\hline$W_{f 2}$ & 1.4 & $W_{S}$ & 8 & $W_{f 2}$ & 1.4 & $W_{S}$ & 8 \\
\hline$W_{f 3}$ & 0.5 & $H_{S}$ & 6 & $W_{f 3}$ & 0.5 & $H_{S}$ & 6 \\
\hline$d_{1}$ & 10 & $S$ & 18 & $d_{1}$ & 10 & $S$ & 18 \\
\hline
\end{tabular}

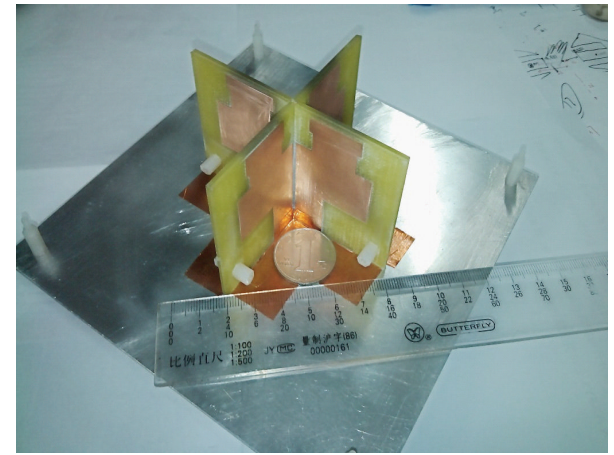

FIGURE 5: The prototype of the broadband dual-polarized antenna.

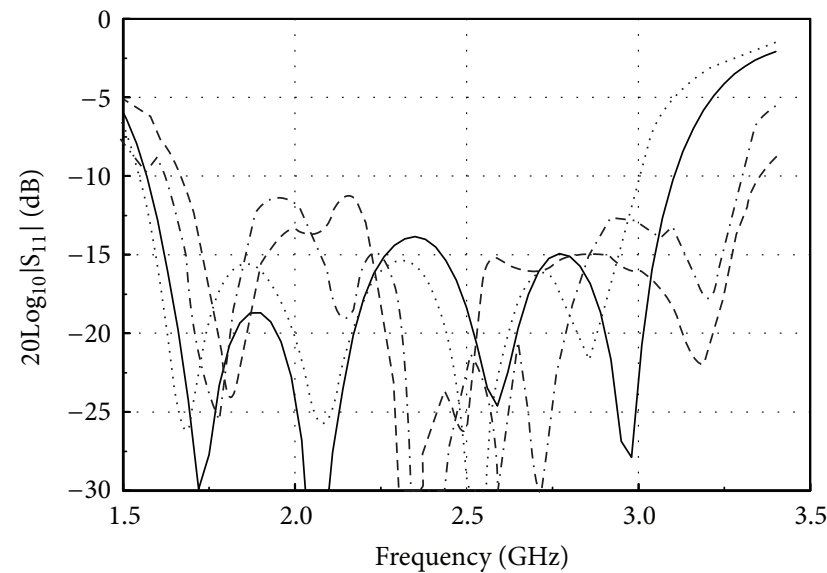

Simulated, port $1 \quad$-.-- Measured, port 1

…. Simulated, port $2 \quad$--- Measured, port 2

Figure 6: Simulated and measured $\left|S_{11}\right|$.

in Figure 7. One can see that the simulated isolation with bending ends is over $5 \mathrm{~dB}$ better than that without bending ends, though the isolation of the measured one seems to have not improved much. The reason is that the coaxial probes of SMA connectors are not properly mounted and connected to the baluns. The minimum measured isolation within the band of $1.5-3 \mathrm{GHz}$ is about $-24 \mathrm{~dB}$. The simulated and measured gains at the two ports are shown in Figure 8. Good agreements are obtained between the simulations and the measurements. Due to the impedance matching at the two

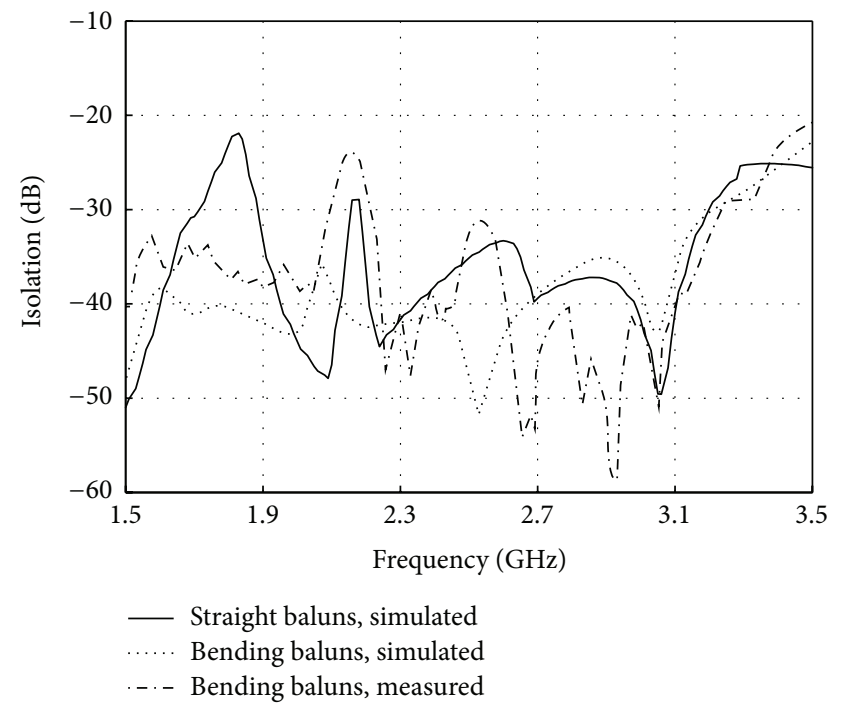

FIgURE 7: Isolation of the two ports.

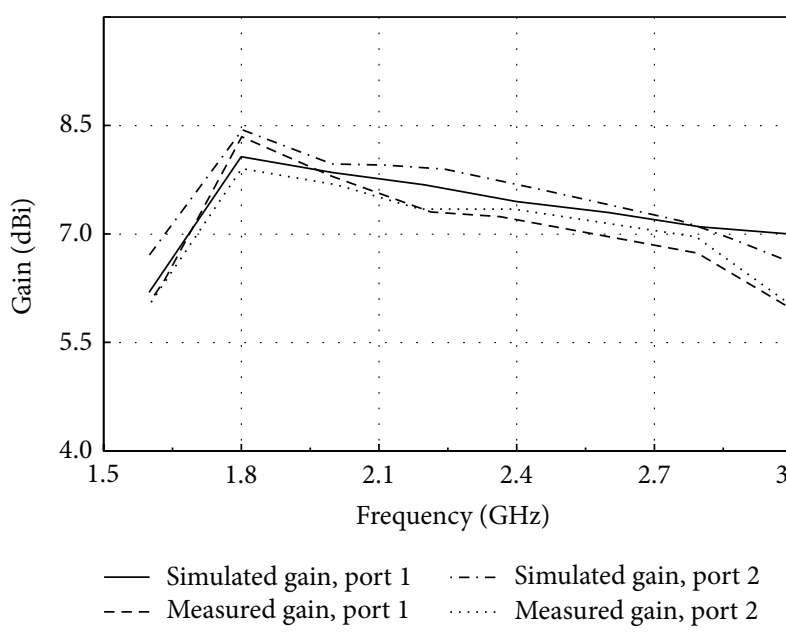

FIGURE 8: Simulated and measured gains at the two ports.

ports, the gains at the two ports increase sharply and the peak gains are achieved at about $1.8 \mathrm{GHz}$ at both ports, where the good impedance matchings are obtained for both ports, as shown in Figure 6 . The measured gain varies among the range of $6-8 \mathrm{dBi}$ within the operating band. 


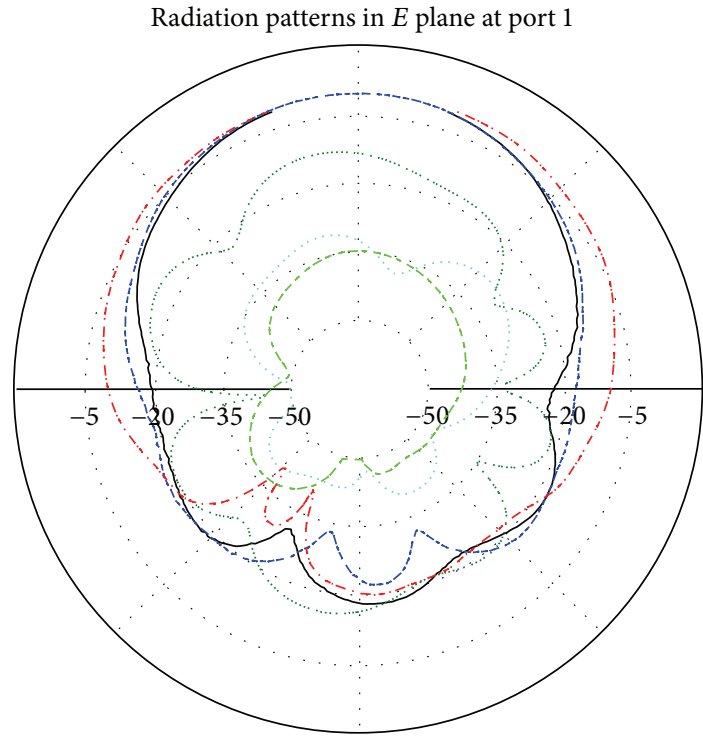

(a)

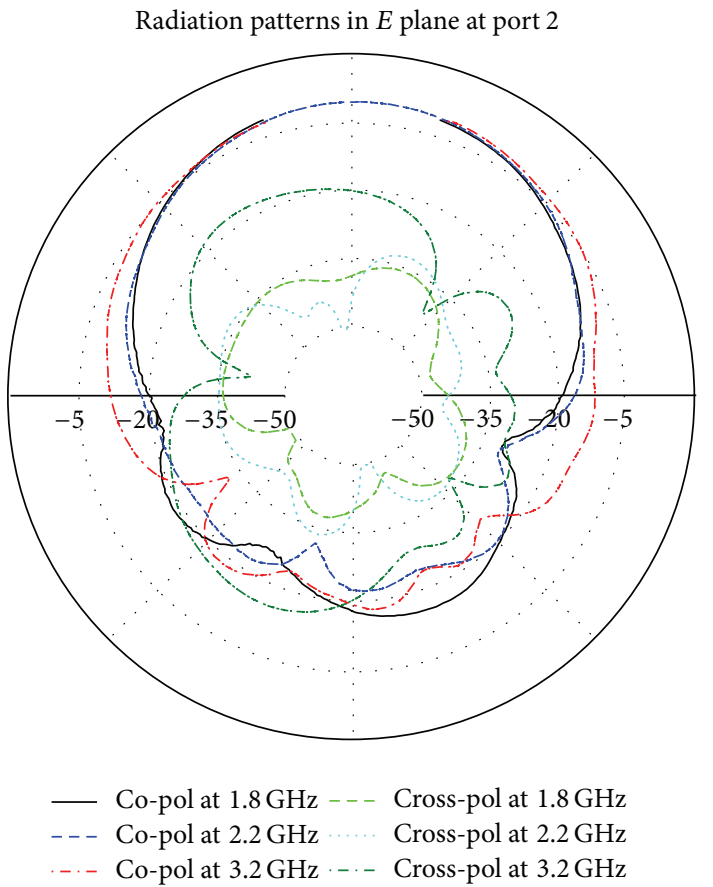

(c)

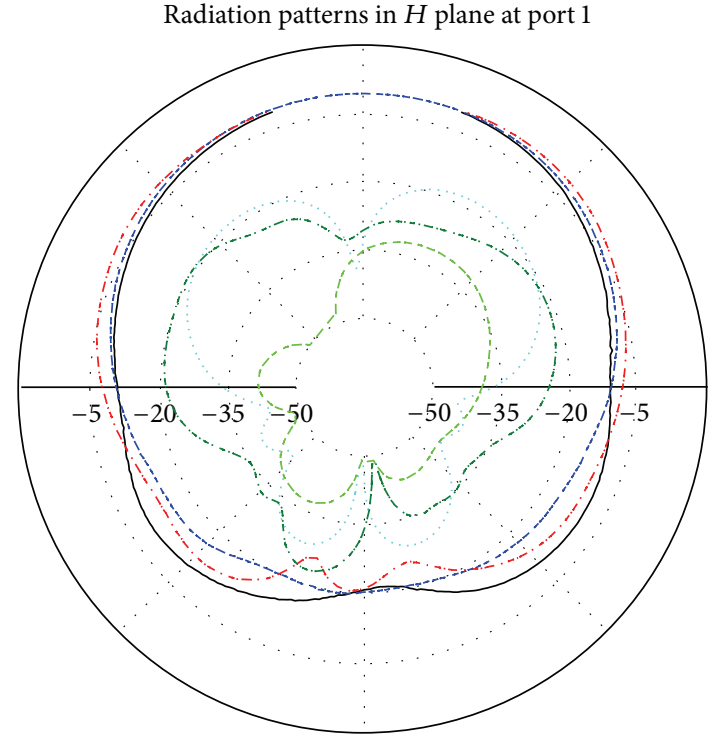

(b)

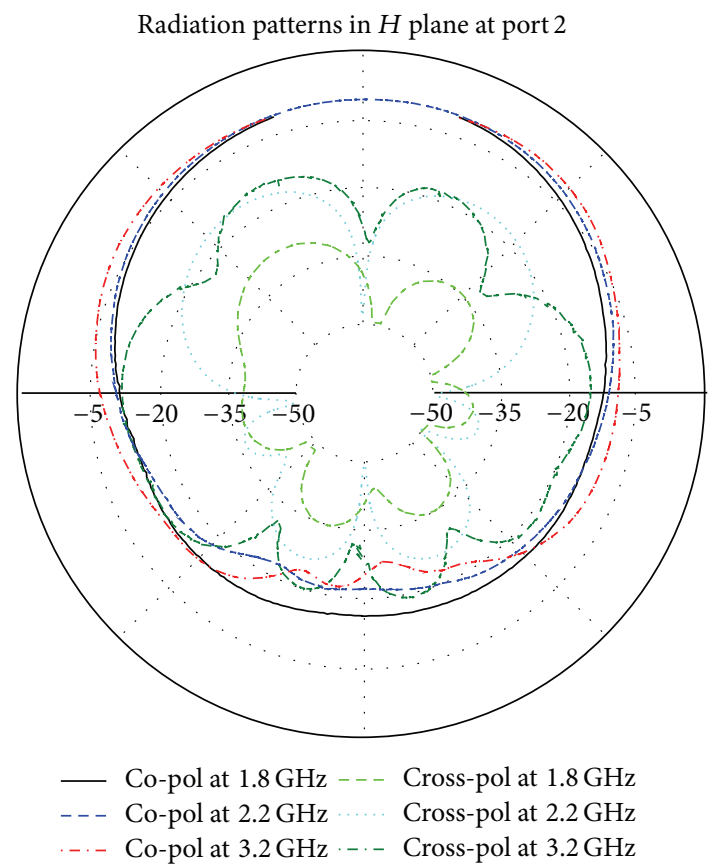

(d)

FIGURE 9: Measured radiation patterns at $1.8 \mathrm{GHz}, 2.2 \mathrm{GHz}$, and $3 \mathrm{GHz}$.

The measured radiation patterns at $1.8 \mathrm{GHz}, 2.2 \mathrm{GHz}$, and 3.2 GHz in E- and $\mathrm{H}$-plane at ports 1 and 2 are shown in Figure 9 , respectively. Reasonable radiation patterns are obtained. As can be seen, the beamwidths at the three frequencies increase with frequency, corresponding to the slow decrease of the gain with frequency. The measured front-to-back ratios are over $12 \mathrm{~dB}$ within the operating band.

\section{Conclusions}

By loading metallic T-shape loadings symmetrically on the upside of the traditional printed dipole, the proposed doublesided printed dipole exhibits a much wider bandwidth. Studies show that the proposed double-sided printed dipole is able to give a bandwidth of over $90 \%$ for $\left|S_{11}\right|<-10 \mathrm{~dB}$. 
Two identical such double-sided printed dipoles and two baluns with different sizes are then cross arranged to form a dual-polarized antenna for TD-LTE base station applications. Due to a PEC ground plane introduced to improve the gain and front-to-back ratio, the dual-polarized design only exhibits a measured bandwidth of $68.8 \%$ for $\left|S_{11}\right|<-10 \mathrm{~dB}$, which also covers the TD-LTE band. The baluns with bending ends will improve the isolation between the two ports and add more separate space for easy mounting and measurement. The antenna is easy to fabricate and can be used as the antenna element of TD-LTE base station antennas.

\section{Conflict of Interests}

The authors declare that there is no conflict of interests regarding the publication of this paper.

\section{Acknowledgments}

This research was supported by the start-up Grants (11BS301) of Huaqiao University, Xiamen, China, the Natural Science Foundation of Fujian Province (2012J01276), China, and Quanzhou Science and Technology Project (Z1424008) from Quanzhou, China.

\section{References}

[1] C. L. Mak, K. M. Luk, K. F. Lee, and Y. L. Chow, "Experimental study of a microstrip patch antenna with an L-shaped probe," IEEE Transactions on Antennas and Propagation, vol. 48, no. 5, pp. 777-783, 2000.

[2] L. Ge and K.-M. Luk, "A wideband magnetio-electric dipole antenna," IEEE Transactions on Antennas and Propagation, vol. 60, no. 11, pp. 4987-4991, 2012.

[3] X.-P. Lu, "Novel broadband printed dipole," Microwave and Optical Technology Letters, vol. 48, no. 10, pp. 1996-1998, 2006.

[4] Z. Zhou, S. Yang, and Z. Nie, "A novel broad-band printed dipole antenna with low cross-polarization," IEEE Transactions on Antennas and Propagation, vol. 55, no. 11, pp. 3091-3093, 2007.

[5] R. Li, T. Wu, B. Pan, K. Lim, J. Laskar, and M. M. Tentzeris, "Equivalent-circuit analysis of a broadband printed dipole with adjusted Integrated balun and an array for base station applications," IEEE Transactions on Antennas and Propagation, vol. 57, no. 7, pp. 2180-2184, 2009.

[6] H.-G. Hao, W.-S. Hu, W. Ruan, and W. Chen, "Miniaturization smart antenna array design for TDSCDMA system," TELKOMNIKA Indonesian Journal of Electrical Engineering, vol. 2, no. 2, 2014.

[7] Q. Zhu, S. Yang, M. Huang, and Z. Nie, "A double-layered printed dipole antenna with parasitic strips," Microwave and Optical Technology Letters, vol. 54, no. 6, pp. 1517-1520, 2012.

[8] B. Q. Wu and K.-M. Luk, "A broadband dual-polarized magneto-electric dipole antenna with simple feeds," IEEE Antennas and Wireless Propagation Letters, vol. 8, pp. 60-63, 2009.

[9] Q. Xue, S. W. Liao, and J. H. Xu, "A differentially-driven dualpolarized magneto-electric dipole antenna," IEEE Transactions on Antennas and Propagation, vol. 61, no. 1, pp. 425-430, 2013.

[10] Y. Cui, R. L. Li, and P. Wang, "A novel broadband planar antenna for 2G/3G/LTE base stations," IEEE Transactions on Antennas and Propagation, vol. 61, no. 5, pp. 2767-2774, 2013. 

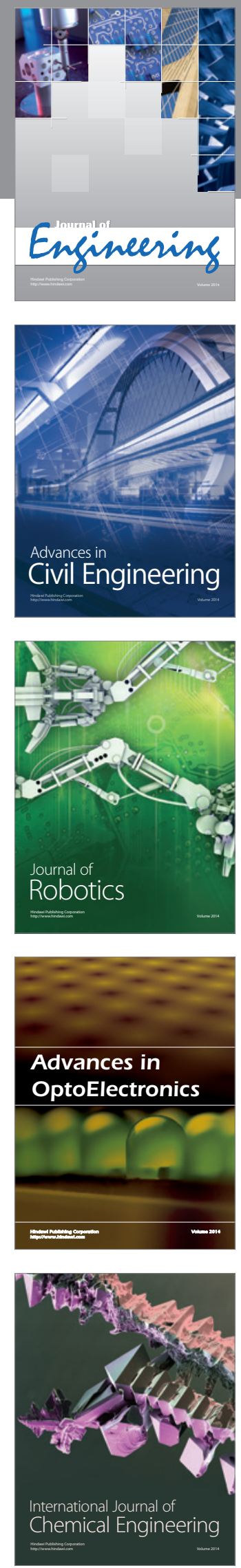

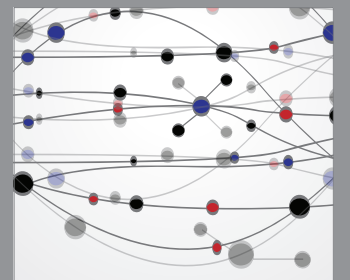

The Scientific World Journal
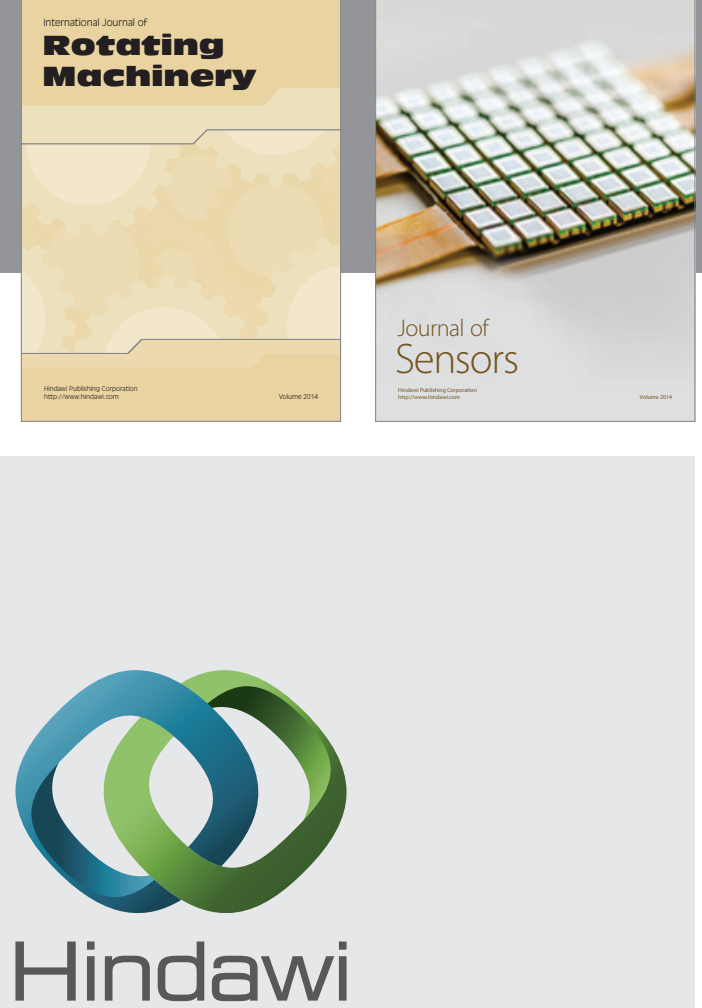

Submit your manuscripts at http://www.hindawi.com
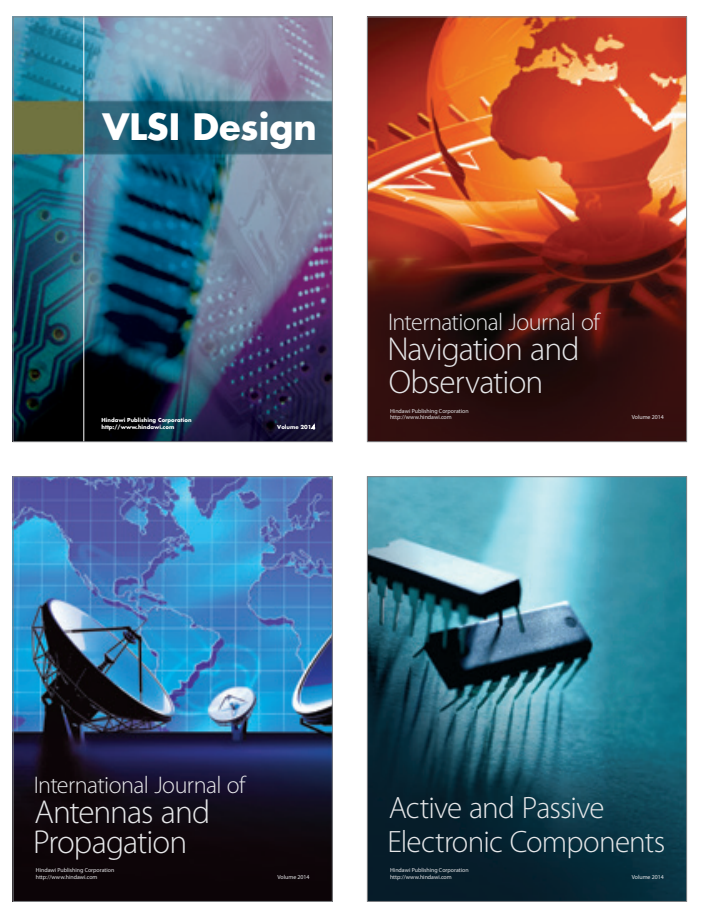
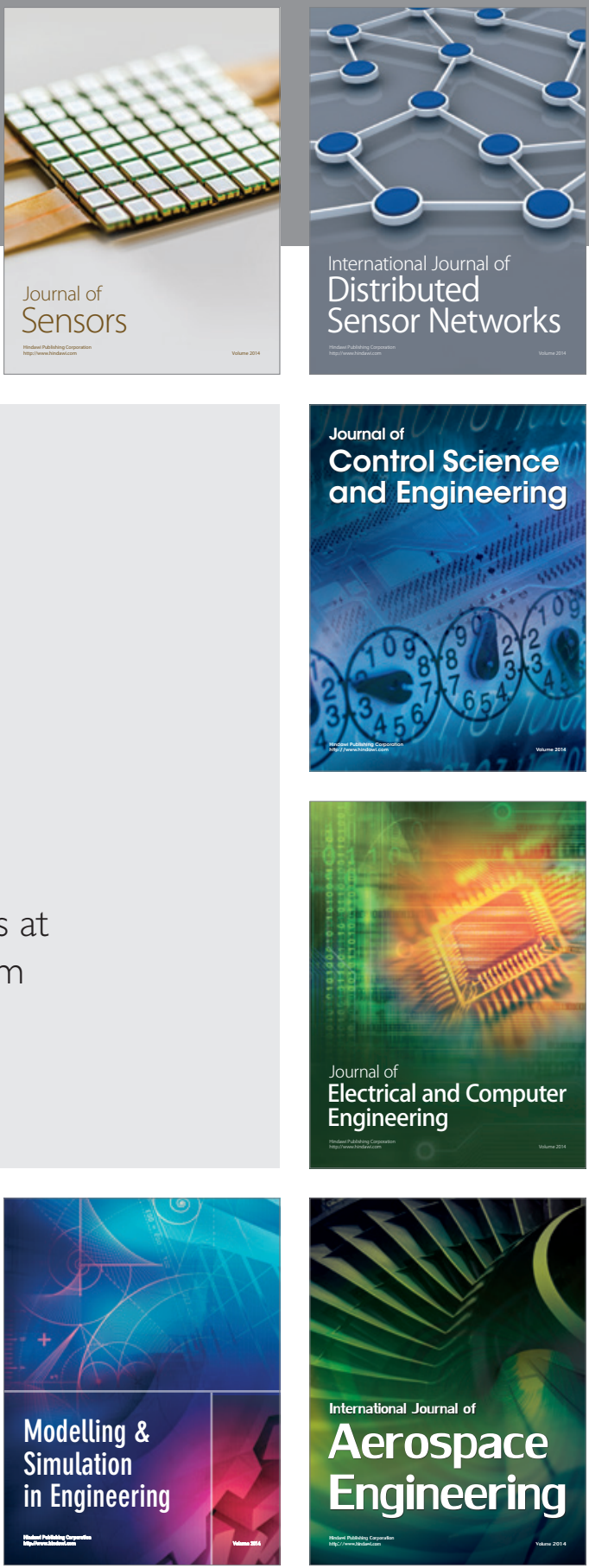

Journal of

Control Science

and Engineering
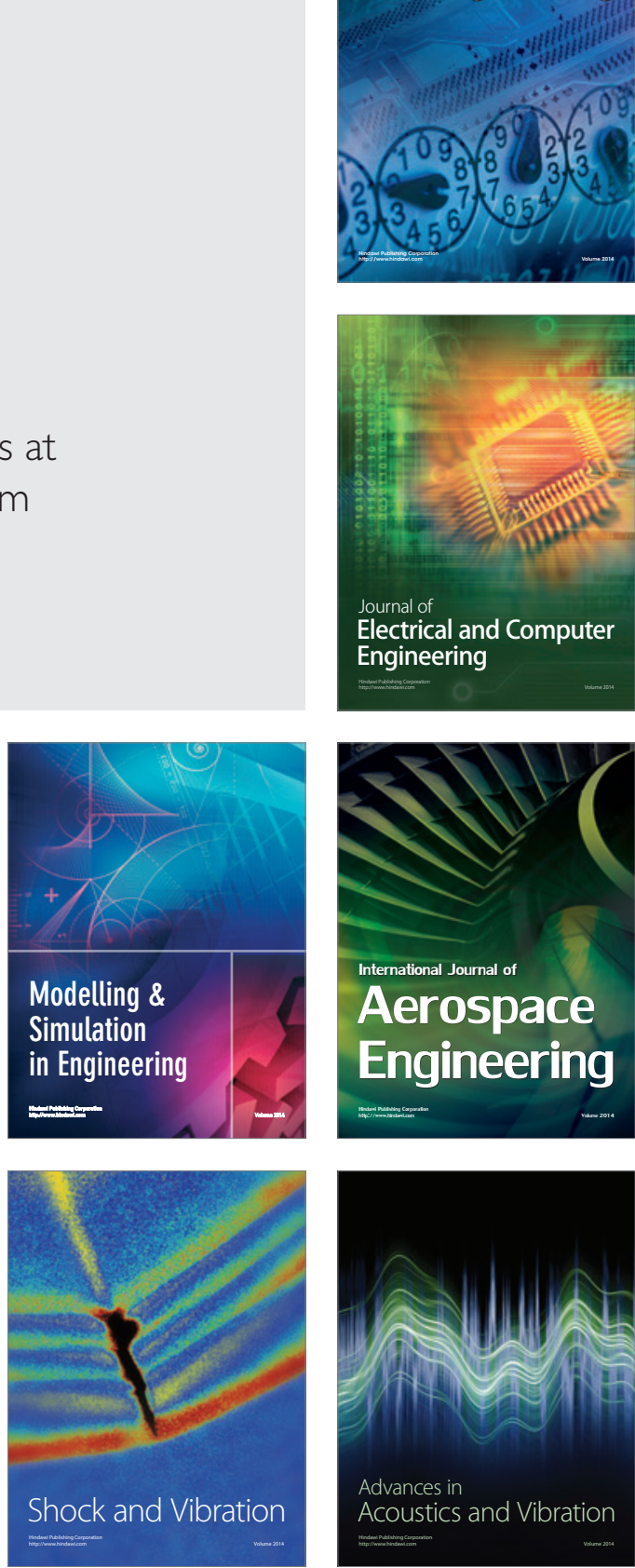